\title{
uma aproximación empírica para calcular la capacidad de los hornos
}

\author{
D. H. GIESKIENG \\ Minerals Processing, n 9, septiembre 1968, págs. 9-11
}

Esta aproximación empírica se basa fundamentalmente sobre el diámetro de la chapa del horno en la zona de clinkerización. Las curvas de capacidad y consumo de combustible frente al diámetro dan las relaciones principales, los resultados de las cuales se relacionan después con la relación longitud/diámetro y otras variables.

Al usar el diámetro de la carcasa en lugar del diámetro interior del ladrillo, surgirá alguna pérdida de calor en la zona de clinkerización, como necesidad práctica que lleva consigo la formación y estabilidad de la costra protectora. Los refractarios en esta zona se han standardizado sobre tipos densos de alta conductividad de calor con placas de acero, conduciendo a la carcasa a una participación activa en el proceso térmico.

En un caso en el que se cambia el refractario de la zona de clinkerización de un horno de $4 \mathrm{~m} \varnothing$ de 15 a $23 \mathrm{~cm}$ de espesor, al reducir el diámetro interior $15 \mathrm{~cm}$, no se afecta de modo apreciable la capacidad. El ladrillo espeso reduce rápidamente su espesor a un valor mas estable de $18 \mathrm{~cm}$.

Sin embargo, al cambiar el diámetro de la carcasa del horno $15 \mathrm{~cm}$, probablemente habrá cambiado su capacidad del orden de un $15 \%$, de acuerdo con la figura 2.

Además, el horno de barlovento en grupos de hornos idénticos tienen normalmente más capacidad que los del sotavento mas calientes. Esto sugiere la posibilidad de encontrar la zona de cocción crítica en la carcasa del horno para encontrar las pérdidas de calor más beneficiosas.

\section{Capacidad del horno convencional}

La figura 1, muestra la relación entre el diámetro de la zona de cocción de un horno convencional y la capacidad, incluyendo los hornos de vía húmeda con cadenas, la vía seca con y sin cadenas, y calentados con carbón, fuel-oil y gas. De la proximidad a la curva media y de la mezcla de datos en las varias condiciones, se deduce que el diámetro en la zona de clinkerización es el factor principal común para valorar la capacidad en el intervalo de tamaños considerado.

Se ha examinado la influencia de la relación longitud/diámetro (L/D) (en un gráfico no representado en este artículo), representando las desviaciones de los datos de la curva media de la figura 1, frente a sus relaciones L/D. Este efecto es, aparentemente, un poco menor del $1 \%$ para $\mathrm{L} / \mathrm{D}$, partiendo de un valor medio $\mathrm{L} / \mathrm{D}$ de 35 . Las tres cuartas partes de los puntos representados en el gráfico caen en un entorno de $\pm 10 \%$ de la 
relación L/D de la curva media y el $90 \%$ de los puntos en un entorno de $\pm 15 \%$, tendiendo a ilustrar la diferencia nominal que actualmente se encuentra en la práctica.

El efecto de la recuperación del calor perdido sobre la capacidad, tal como ocurre con los sistemas de cadenas, es aparentemente proporcional al porcentaje de calor ahorrado en relación con la marcha sin estos dispositivos, y a que entonces se requiere mucha menos proporción de calor en la zona de clinkerización crítica por unidad de producción. Por ejemplo, dos hornos de $3 \mathrm{~m}$ de $\varnothing$ (1) y (2) representados en la figura 1 , con la misma alimentación y la misma longitud: el (1) está sin cadenas y consume $1.330 \mathrm{kcal} / \mathrm{kg}$, mientras que el (2) tiene un amplio sistema de cadenas y cruces y un consumo de combustible de $965 \mathrm{kcal} / \mathrm{kg}$.

La curva de la figura 1 es, en efecto, una media de la práctica actual, donde un horno de vía seca sin cadenas puede tener quizás una mayor facilidad de cocción que un horno idéntico con un pequeño sistema de cadenas, y ambos, por lo tanto, pueden tener capacidades similares.

\section{Sistemas de cadenas}

Mas que concentrar todo el sistema de cadenas próximo al extremo de la alimentación en un horno de vía seca, por un esfuerzo de reducir su exposición a las más altas temperaturas, parece más conveniente tener en cuenta la ventaja de emplear aleaciones de más resistencia al calor y distribuir la misma cantidad de cadenas a lo largo del horno hasta la máxima temperatura permisible. Esto ampliaría la agitación del lecho hasta zonas más bajas del horno creando una exposición adicional del material, cadenas y refractarios a gases más calientes. Asimismo, al aumentar la distancia media del sistema de cadenas del extremo de la alimentación, permite que se deposite más polvo antes de que los gases abandonen el horno.

Puesto que una gran parte del mecanismo de transmisión de calor entre los gases, las cadenas y el material, en la vía seca, tiene lugar por radiación, es de esperar que la temperatura más elevada de trabajo obtenida mejorará el comportamiento en una cantidad aproximada de:

$$
\left(\frac{T_{a b s}}{100}\right)^{4} \text {. }
$$

Esto es la cuarta potencia de la energía: un pequeño aumento en la temperatura dará una réplica amplia.

El mismo principio se aplica a un horno de vía húmeda. Una vez seco el material, parece que las cadenas restantes en el sistema pueden diluirse en su distribución a medida que se profundiza en el horno.

\section{Capacidad del horno con precalentador en suspensión gaseosa}

La figura 2 muestra la relación que existe entre el diámetro de la chapa del horno en la zona de clinkerización y la capacidad de hornos con precalentadores en suspensión gaseosa calentados con carbón y fuel-oil. Para derivar el efecto L/D, se representan las desviaciones de la curva media frente a las variaciones de L/D de cada horno (fig. 3). Esta curva es la base al introducir una variable muy importante: el efecto no lineal de las relaciones $\mathrm{L} / \mathrm{D}$ en el horno con precalentador. 
La figura 4 contiene los puntos de la figura 2, corregida por la curva de la figura 3 . Es evidente una concordancia mejorada si se compara con la figura 2. Las desviaciones que subsisten pueden deberse a: 1) química y tamaño de la alimentación; 2) influencia relativamente menor de elementos auxiliares tales como molino de carbón e influencia del clínker; 3) pérdida de polvo; y 4) exactitud de los datos.

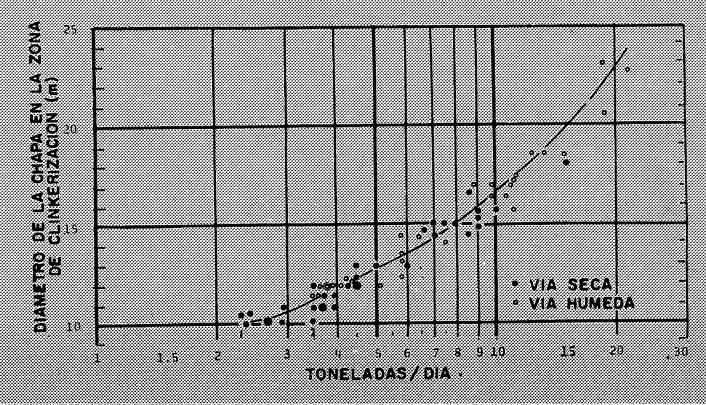

Fig. 1.-Capacidades de hornos convencionales en t/día para una relación $L / D$ no corre-

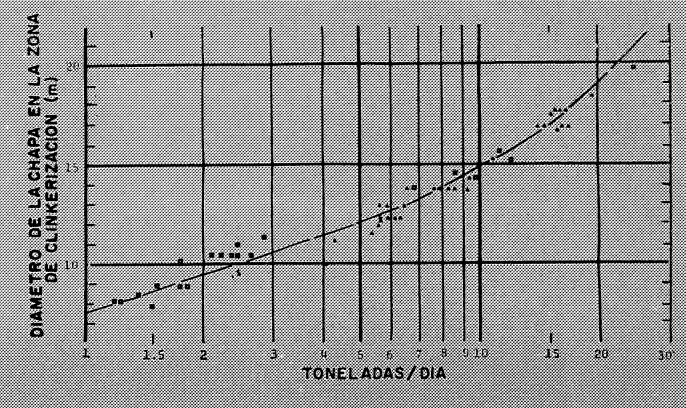

Fig. 2.-Capacidades de hornos con precalentador en $t /$ día, para relaciones $L / D$ o aptitu

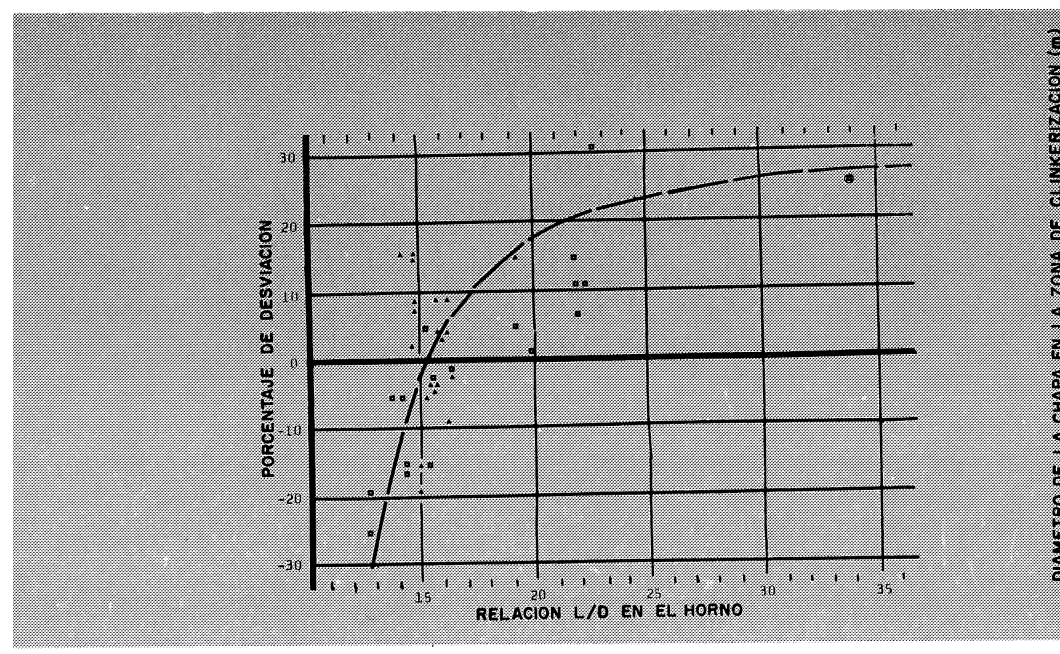

Fig. 3.- Horno con precalentador con la curva de corrección L/D.

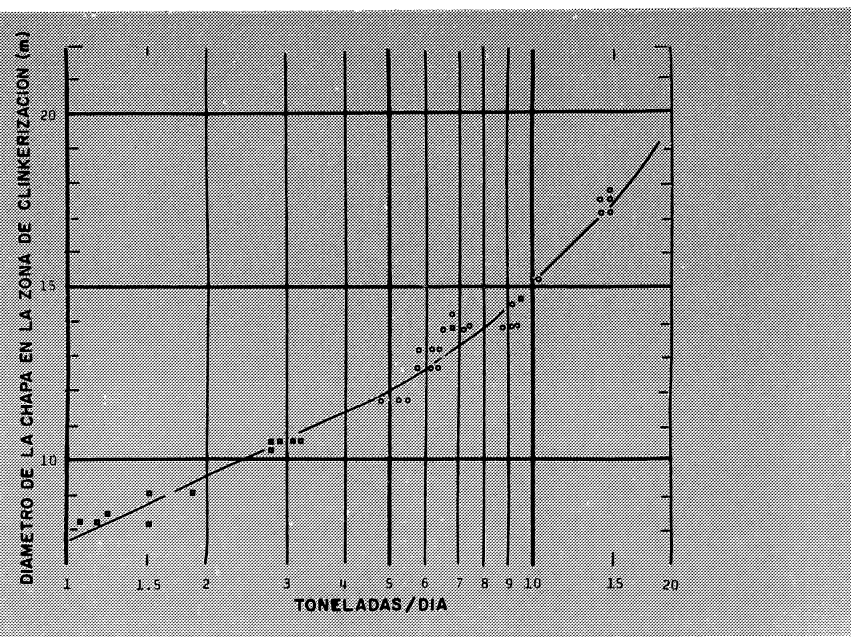

Fig. 4. - Capacidades de horno con precalentador
en $t / d i ́ a$, corregidas para la relación $L / D$.

\section{Aptitud a la cocción - precalentadores de ciclón}

No se dispone de amplios datos sobre la química y finura de la alimentación. Pero la matriz de la figura 5 se ha formado sobre la base de seis puntos observados que abarcan la mayor parte del intervalo práctico de saturación en cal y finura. La figura 6 muestra donde se encontraban estos puntos en la figura 4 y las pequeñas flechas indican la posición resultante cuando se emplean las correciones de actitud a la clinkerización sugeridas por la figura 5. 
Aunque las tendencias sugeridas por la figura 5, deben de comprobarse más adelante, las indicaciones inclinan a creer que la química de la alimentación y el tamaño son los factores dominantes en la desviación que subsiste en la figura 4, junto con factores tales como el equipo auxiliar, aunque de una importancia mucho menor.

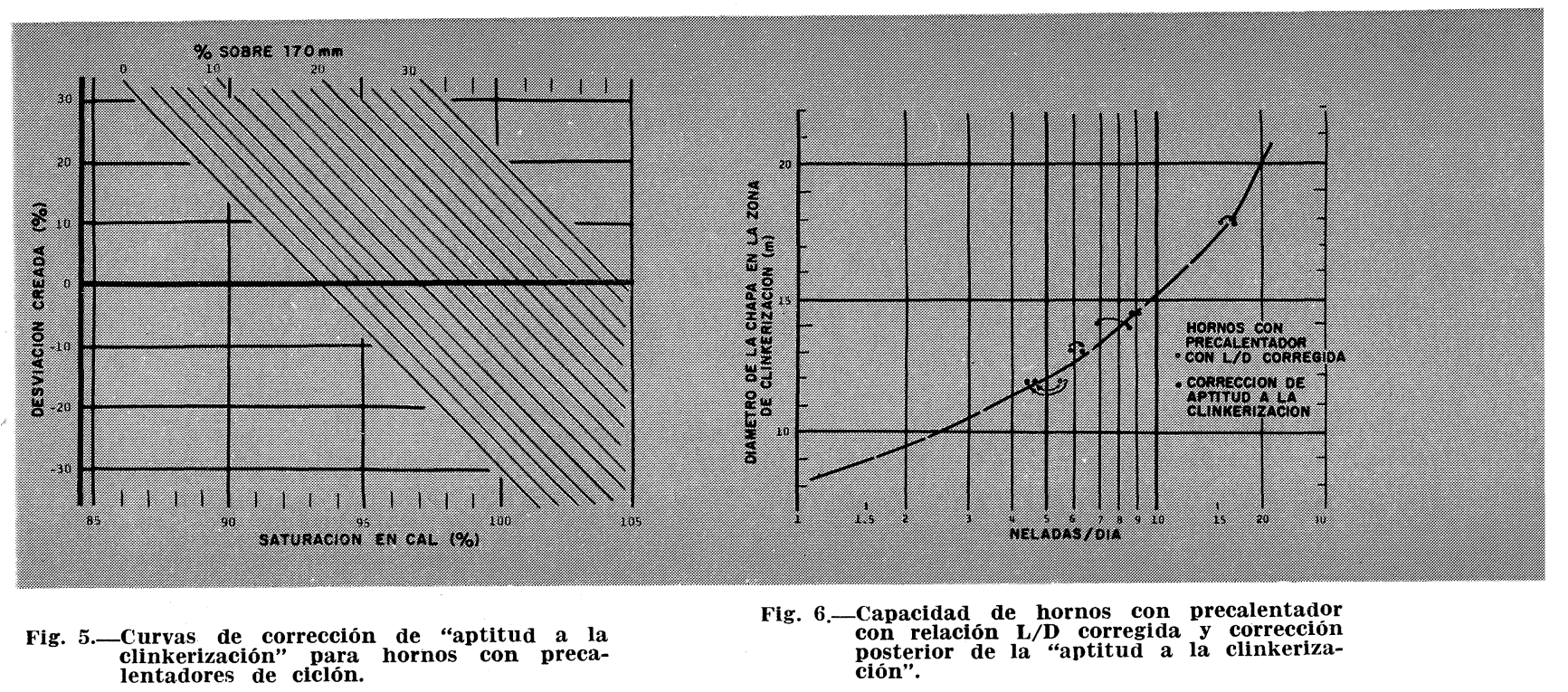

\section{Aptitud a la cocción - hornos convencionales}

Debido a las desviaciones muy nominales con las que se empieza en la figura 1 y a la falta de datos completos, no ha sido posible formular una curva capacidad/química-tamaño para hornos convencionales. Puesto que una alimentación más fina en algunos hornos convencionales se ha considerado como una mejora de la capacidad, este efecto puede ser despreciable o aún opuesto al subrayado en la figura 5, cuando los hornos están equipados con ciclones precalentadores en suspensión gaseosa.

\section{Consumo de combustible de los hornos con precalentador}

A causa de la importancia de las pérdidas por radiación de la chapa en la zona de clinkerización del horno, su diámetro tiene un efecto importante sobre el consumo de combustible (fig. 7). Las desviaciones de la curva media son de un porcentaje discreto bastante bajo, como para hacer impracticable intentar distinguir entre polvo, efectos que causa la química-tamaño de alimentación, continuidad y exactitud de datos. Sin embargo, parece discernible el efecto de la relación L/D sobre las desviaciones de combustible, como se muestra en la figura 8.

La revisión de las figuras 3 y 8 indican la posibilidad de desear un alargamiento de los hornos equipados con precalentador, a una relación L/D de por ejemplo 20 , en vez de 15 a 16 como es normal actualmente. Se gana algo de capacidad y el consumo de combustible es aparentemente tan bajo o ligeramente inferior. Esto parece deberse a los beneficios obtenidos con un tiempo de retención adicional que facilita un tratamiento completo en la zona de calcinación y un comportamiento aceptable del horno a los distintos niveles de temperatura en toda su longitud. 


\section{Consumo de combustible - hornos convencionales}

A medida que se añaden cadenas a los hornos de vía seca convencionales, se rebaja el consumo de combustible a valores del mismo orden que los logrados con los precalentadores en supensión: diversos hornos han registrado $965 \mathrm{kcal} / \mathrm{kg}$ o menos.

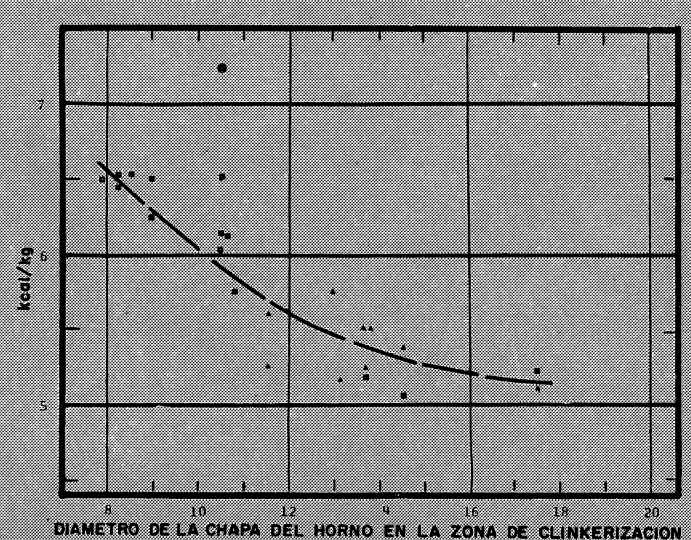

Fig. 7.-Consumo de combustible, en kcal/kg, de hornos con precalentador en función del
diámetro de la chapa del horno en la diámetro de la chapa
zona de clinkerización.

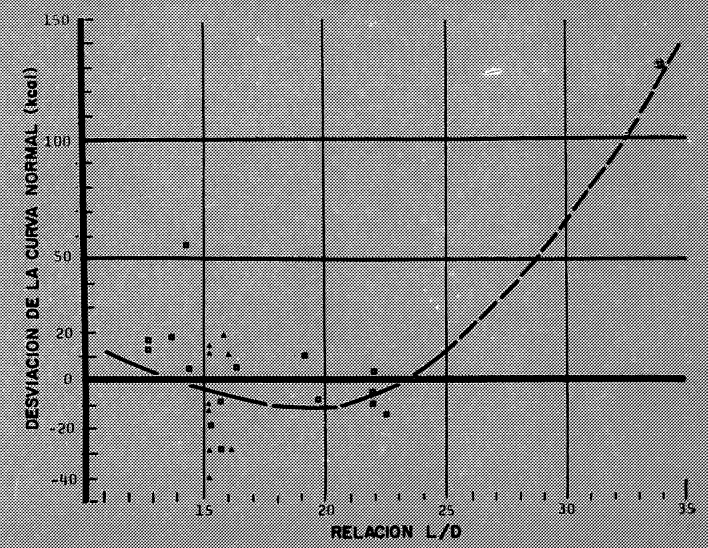

Fig. 8.-Desviaciones de la norma, consumo de combustible de relación $\mathbf{L} / \mathbf{D}$.

\section{Curvas frente a ecuaciones}

Puesto que la mayor parte de las curvas presentadas no son fáciles de deducir a ecuaciones exactas y sencillas, no se ha intentado realizar este trabajo. Realmente se perdería exactitud sin conseguir grandes ventajas. Las curvas además proporcionan una ayuda visual para reconocer el campo de desviación y las probabilidades de adaptación.

\section{Resumen y mirada retrospectiva}

El análisis hecho en este breve trabajo identifica por separado la limitación de factores en las varias areas del proceso en el horno de cemento, principalmente las zonas de preparación y clinkerización. Esto da un conocimiento mas útil y real del proceso, si se compara con el intento de atacar las características del horno con un criterio empírico como es el volumen interno. 